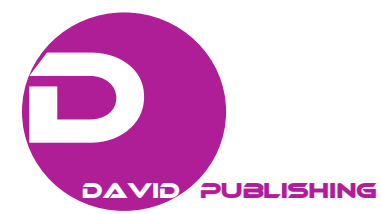

\title{
Isogeometric Analysis for Linear and Nonlinear Cantilever in CAD
}

\author{
Mian Zhou \\ Department of Machine and Process Engineering, Technical University Kaiserslautern, Kaiserslautern 67655, Germany
}

\begin{abstract}
A novel method for the mechanical simulation of linear and nonlinear Timoshenko-beams has been presented. The beam strains are based on a kinematic assumption where the shear deformation and rotational are considered. Applying the isoparametric concept the kinematic quantities are approximated using NURBS (non-uniform Rational B-spline) functions. This numerical simulation can be called as isogeometric analysis, which can improve the efficiency in CAD (computer aided design). Furthermore, an efficient Code has been developed and the results for two numerical applications are given in the end.
\end{abstract}

Key words: Isogeometric analysis, NURBS basis functions, Timoshenko-beams, finite element formulation.

\section{Introduction}

Three-dimensional beam-like structures can be found frequently in man-made structures, like helicopter blades, robot arms or rotor blades. Due to the shear deformation and rotational bending effects, Stephen Timoshenko introduced the so-called Timoshenko-beam theory early in the 20th century to describe the behavior of this beams [1]. According to the Timoshenko-beam theory, several authors developed finite element formulations for three-dimensional beams using a Lagrangian formulation, see Bathe and Bolourchi [2]. The problem of this formulation is imprecise approaches of the geometry of the three-dimensional beam in CAD and analysis. Furthermore this kind of formulation increases the computational cost.

Currently we can solve this problem with the help of the isogeometric analysis, which is developed by J. Austin Cottrell and Thomas J. R. Hughes in 2005. The isogeometric analysis is the bridge between integrating FEA (finite element analysis) and NURBS (non-uniform rational B-spline) based CAD (computer aided design). The basic idea of isogeometric analysis

Corresponding author: MianZhou, Master of Science, research field: computational engineering. is to use the same geometry description for design and analysis. Also there are many computational technologies that may be used in isogeometric analysis, e.g. NURBS or T-splines. Due to the convenience of the free form surface modeling the NURBS-functions are chosen as usual. The isogeometric analysis has been provided by many authors (e.g. Morganti [3]), that the isogeometric analysis can increase the accuracy and reduce the computational cost.

In this paper the isogeometric analysis for linear and nonlinear cantilever is discussed. A cantilever is a basic structural element in industrial design, e.g. beam or plate, which can be considered as three-dimensional beam. Furthermore the author developed an efficient Code in order to calculate the deflection of the cantilever under different loads.

\section{Isogeometric Parameterization of the Beam Model}

\subsection{NURBS Basis Functions and Curves}

In Computational Engineering B-spline and NURBS are commonly used for describing curves and surfaces because of its great flexibility and precision. Here we briefly discuss the definitions and properties of NURBS, which can be found in Refs. [4, 5]. 
Before talking about the B-spline we define a knot vector $\Xi=\left\{\xi_{1}, \xi_{2}, \ldots, \xi_{n+p+1}\right\}$, which is non-decreasing sequence of knots $\xi_{i} \in \mathbb{R}$ on the parameter space. $i$ is the knot index from 1 to $n+p+1$, where $p$ is the polynomial order and $n$ is the number of basis functions. If the knots are divided equidistantly $\left(\xi_{i}-\xi_{i+1}=\right.$ constant $)$ into parameter space, the knot vector is defined as uniform, otherwise it is called non-uniform. Usually we discuss the open knot vector, which the first and last knot values repeat $p+1$ times

$$
\begin{gathered}
(\Xi= \\
\left.\{\underbrace{\xi_{1}, \xi_{2}, \ldots, \xi_{p+1}}_{p+1}, \ldots, \xi_{n-1}, \xi_{n}, \underbrace{\xi_{n+1}, \xi_{n+2}, \ldots, \xi_{n+p+1}}_{p+1}\}\right) .
\end{gathered}
$$

The B-spline basis functions $N_{i, p}(\xi)$ are defined by the Cox-de Boor recursion formula (Cox, 1971; de Boor, 1972). If $p=0$ :

$$
N_{i, 0}(\xi)=\left\{\begin{array}{l}
1, \xi_{i} \leq \xi<\xi_{i+1} \\
0, \text { else }
\end{array}\right.
$$

If $p>0$ :

$$
\begin{aligned}
N_{i, p}(\xi)=\frac{\xi-\xi_{i}}{\xi_{i+p}-} & \xi_{i} \\
& N_{i, p-1}(\xi) \\
& +\frac{\xi_{i+p+1}-\xi}{\xi_{i+p+1}-\xi_{i+1}} N_{i+1, p-1}
\end{aligned}
$$

Caution: The quotient $\%$ is defined to be 0 .

Furthermore we introduce some important properties for B-spline, which are usually used.

Partition of unity: $\sum_{i=0}^{n} N_{i, p}(\xi)=1$, for all $\xi$;

Nonnegativity: $N_{i, p}(\xi) \geq 0, \forall \xi$;

Derivatives: Each $p^{\text {th }}$ order function has $\mathrm{p}-$ 1 continuous derivatives across the boundaries.

Next we introduce vector-valued coefficients $B_{i}$, which are called control points. With help of those we can build a B-spline curve easily.

$$
\mathrm{C}(\xi)=\sum_{i=1}^{n} N_{i, p}(\xi) B_{i}
$$

The difference between B-spline basis functions $N_{i, p}$ and NURBS basis functions $R_{i, p}$ is additional weight $\mathrm{s} \omega_{i}$, which is ever positive.

$$
R_{i, p}=\frac{N_{i, p}(\xi) \omega_{i}}{\sum_{j=1}^{n} N_{j, p}(\xi) \omega_{j}}
$$

Analog a NURBS curve can be written as

$$
\mathrm{C}(\xi)=\sum_{i=1}^{n} R_{i, p}(\xi) B_{i} .
$$

In the end some important properties of spline curves on open knot vectors will be mentioned.

Convex hull property: The curve is completely contained in its control polygon.

Locality: $N_{i, p}(\xi)=0$, if $\xi \notin\left[\xi_{i}, \xi_{i+p+1}\right)$;

Interpolation of start and end points: $\mathrm{C}\left(\xi_{1}\right)=C_{1}$, $\mathrm{C}\left(\xi_{m}\right)=C_{n}$.

Affine transformations of the curve can be performed directly on its control points.

\subsection{Parameterization of Linear Timoshenko-Beam}

One of the simplest and most important design elements is the beam, which has a ready application in engineering work. There are two assumptions of beam, which should be mentioned. The one is "plane sections remain plane", which means, the plane cross-sections of the undeformed beam remain plane to the deflection curve of the deformed beam. Another is that, the deformation of the beam in the vertical direction should be neglected. In this paper we discuss the Timoshenko-beams, which occur in different areas of engineering practice.

According to the technical mechanics [6] we can write the following basic equations easily.

$$
\begin{aligned}
\omega=\omega(x) u(x, z) & =-z \beta(x) \gamma_{x z}=\frac{\partial \omega}{\partial x}+\frac{\partial u}{\partial z} \epsilon \\
& =\frac{\partial u}{\partial x}=-z \beta^{\prime} \gamma=\omega^{\prime}-\beta
\end{aligned}
$$

For linear Timoshenko-beam the transverse force $Q$ and the bending moment $M$ can be written as:

$$
Q=\int \tau d A=G \bar{A} \gamma M=\int z \sigma d A=-E I \beta^{\prime} .
$$

Here $\bar{A}$ is the cross-section due to the shear stress. Furthermore the deflection and the Twist can be 
written as $\omega$ and $\beta$. Now the potential of internal and external forces can be presented as following:

$$
\begin{gathered}
\prod_{i}[\omega, \beta]=\frac{1}{2} \int_{0}^{l}\left(M \beta^{\prime}+Q \gamma\right) d x \prod_{e}[\omega, \beta] \\
=-\int_{0}^{l} q \omega d x
\end{gathered}
$$

According to the principle of virtual work the total potential is zero.

$$
\begin{aligned}
\delta\left(\prod_{i}[\omega, \beta]+\prod_{a}[\omega, \beta]\right) & \\
= & \int_{0}^{l} E I \beta^{\prime} \delta \beta^{\prime} d x \\
& +\int_{0}^{l} G \bar{A}\left(\omega^{\prime}-\beta\right)\left(\delta \omega^{\prime}-\delta \beta\right) d x \\
& -\int_{0}^{l} q \delta \omega d x=0
\end{aligned}
$$

$$
\delta \Pi^{e}=\left(\delta \boldsymbol{u}^{e}\right)^{T}\{[\underbrace{\int_{0}^{e} \boldsymbol{B}_{\beta}^{e T} E I \boldsymbol{B}_{\beta}^{e} d x^{e}+\int_{0}^{l e}\left(\boldsymbol{B}_{\omega}^{e T}-\boldsymbol{N}_{\beta}^{e T}\right) G A_{s}\left(\boldsymbol{B}_{\omega}^{e}-\boldsymbol{N}_{\beta}^{e}\right) d x^{e}}_{\boldsymbol{K}^{e}}] \boldsymbol{u}^{e}-\underbrace{\int_{0}^{l e} \boldsymbol{N}_{\omega}^{e T} \boldsymbol{q} d x^{e}}_{\boldsymbol{f}^{e}}\}
$$

Beside, the matrix $\boldsymbol{K}^{e}$ and the vector $\boldsymbol{f}^{e}$ denote the tangential stiffness matrix and sum of the internal and external forces.

$$
\boldsymbol{K}^{e} \boldsymbol{u}^{e}=\boldsymbol{f}^{e}
$$

\subsection{Parameterization of Nonlinear Timoshenko-Beam}

The finite element formulation of nonlinear Timoshenko-beam is different from the linear situation. First of all we introduce an orthogonal basis system $\boldsymbol{A}_{i}$ of reference configuration and $\boldsymbol{a}_{i}$ of current configuration. Therefore we can describe the axis of the beam along $\boldsymbol{A}_{1}$ with the arc-length parameter $S=\xi_{1} \in[0, L]$. The cross-sections of the beam are described by the local coordinates $\left\{\xi_{2}, \xi_{3}\right\}$, which is shown in Fig. 1.

Hence, the position vectors of the undeformed and deformed cross-sections can be expressed as

$$
\begin{gathered}
\boldsymbol{X}\left(\xi_{2}, \xi_{3}, S\right)=\boldsymbol{X}_{0}(S)+\xi_{2} \boldsymbol{A}_{2}(S)+\xi_{3} \boldsymbol{A}_{3}(S) \\
\boldsymbol{x}\left(\xi_{2}, \xi_{3}, S, t\right)=\boldsymbol{x}_{0}(S, t)+\xi_{2} \boldsymbol{a}_{2}(S, t)+\xi_{3} \boldsymbol{a}_{3}(S, t)
\end{gathered}
$$

$\boldsymbol{A}_{i}$ and $\boldsymbol{a}_{i}$ are the vectors, which follow from the
Next we can approximate Eq. (9) with finite element. According to the isoparametric concept, the following kinematic variables are interpolated with NURBS shape functions $N_{I}(\xi)$.

$$
\begin{aligned}
\omega^{e} & =\left[\begin{array}{lllllll}
N_{1}^{e} & 0 & N_{2}^{3} & 0 & \ldots & N_{\text {nen }}^{e} & 0
\end{array}\right] \boldsymbol{u}^{e} \\
\beta^{e} & =\left[\begin{array}{lllllll}
0 & N_{1}^{e} & 0 & N_{2}^{e} & \ldots & 0 & N_{\text {nen }}^{e}
\end{array}\right] \boldsymbol{u}^{e} \\
\delta \omega^{e} & =\left[\begin{array}{lllllll}
N_{1}^{e} & 0 & N_{2}^{3} & 0 & \ldots & N_{\text {nen }}^{e} & 0
\end{array}\right] \delta \boldsymbol{u}^{e} \\
\delta \beta^{e} & =\left[\begin{array}{lllllll}
0 & N_{1}^{e} & 0 & N_{2}^{e} & \ldots & 0 & N_{\text {nen }}^{e}
\end{array}\right] \delta \boldsymbol{u}^{e}
\end{aligned}
$$

Hereby the number of the element in the columns matrix

$$
\boldsymbol{u}^{e}=\left[\begin{array}{lllllll}
\omega_{1}^{e} & \beta_{1}^{e} & \omega_{2}^{e} & \beta_{2}^{e} & \ldots & \omega_{n e n}^{e} & \beta_{\text {nen }}^{e}
\end{array}\right] \text { is }
$$
dependent on the control points of NURBS-functions. Furthermore, $\omega^{e^{\prime}}, \beta^{e^{\prime}}, \delta \omega^{e^{\prime}}$ and $\delta \beta^{e^{\prime}}$ are given replacing $N_{I}$ by $N_{I}^{\prime}$ in Eqs. (10)-(13). Thus, Eq. (9) can be expressed as follows

orthogonal transformations $\left(\boldsymbol{A}_{i}=\boldsymbol{R}_{0} \boldsymbol{e}_{i}, \boldsymbol{a}_{i}=\boldsymbol{R e}_{i}\right)$. The matrix $\boldsymbol{R}$ follows from the so-called Rodrigues formula. Applying the following well-known Euler-Lagrange equilibrium equations of a three-dimensional beam

$$
\begin{gathered}
\boldsymbol{f}^{\prime}+\overline{\boldsymbol{n}}=0 \\
\boldsymbol{m}^{\prime}+\boldsymbol{x}_{0}^{\prime} \times \boldsymbol{f}+\overline{\boldsymbol{m}}=0
\end{gathered}
$$

We obtain the virtual work that can be expressed as follows

$$
\begin{array}{r}
\mathcal{G}(\boldsymbol{v}, \delta \boldsymbol{v})=-\int_{S}\left[\left(\boldsymbol{f}^{\prime}+\overline{\boldsymbol{n}}\right) \cdot \delta \boldsymbol{x}_{0}+\left(\boldsymbol{m}^{\prime}+\boldsymbol{x}_{0}^{\prime} \times \boldsymbol{f}+\right.\right. \\
\boldsymbol{m} \cdot \delta \boldsymbol{w} d S=0 .
\end{array}
$$

Now we introduce two strain measures $\varepsilon$ and $\kappa$, which have been proposed by Reissner [7].

$$
\boldsymbol{\varepsilon}=\boldsymbol{R}^{T} \boldsymbol{x}_{0}^{\prime}-\boldsymbol{R}_{0}^{T} \boldsymbol{X}_{o}^{\prime} \boldsymbol{\kappa}=\boldsymbol{R}^{T} \boldsymbol{\theta}-\boldsymbol{R}_{0}^{T} \boldsymbol{\theta}_{0}
$$

The variations of those strain measures are given as follows:

$$
\begin{gathered}
\delta \boldsymbol{\varepsilon}=\boldsymbol{R}^{T} \delta \boldsymbol{x}_{0}^{\prime}+\delta \boldsymbol{R}^{T} \boldsymbol{x}_{0}^{\prime}=\boldsymbol{R}^{T}\left(\delta \boldsymbol{x}_{0}^{\prime}-\delta \boldsymbol{w} \times \boldsymbol{x}_{0}^{\prime}\right) \\
\delta \boldsymbol{\kappa}=\boldsymbol{R}^{T} \delta \boldsymbol{\theta}+\delta \boldsymbol{R}^{T} \boldsymbol{\theta}=\boldsymbol{R}^{T} \delta \boldsymbol{w}^{\prime}
\end{gathered}
$$

A proof of Eq. (23) is given in Ref. [8]. With $\mathbf{f}=\mathbf{R F}$ and $\mathbf{m}=\mathbf{R} \mathbf{M}$ the virtual work can be 

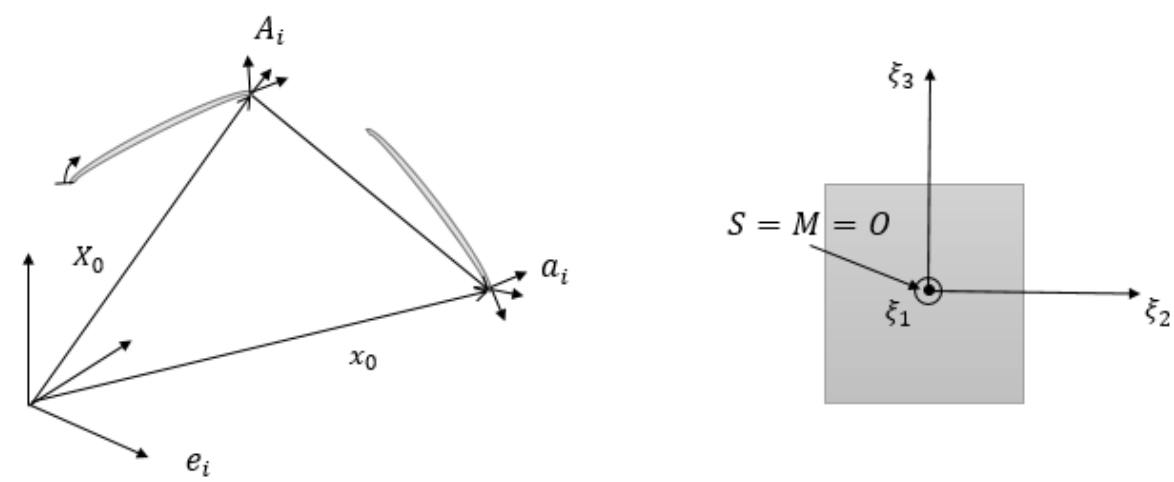

Fig. 1 Reference and current configuration of the Timoshenko-beam.

reformulated using the relationship $\mathrm{d} V=\mathrm{d} A \mathrm{~d} S$. This yields

$$
\begin{aligned}
\mathcal{G}(\boldsymbol{v}, \delta \boldsymbol{v})=\int_{s}( & \left.\delta \widehat{\boldsymbol{E}}^{T} \widehat{\boldsymbol{S}}-\overline{\boldsymbol{n}} \cdot \delta \boldsymbol{x}_{q}\right) d S \\
& =\int_{\Omega_{0}} \boldsymbol{S} \cdot \delta \boldsymbol{E} d V \\
& -\int_{\Omega_{0}} \rho_{0} \overline{\boldsymbol{b}} \cdot \delta \boldsymbol{u} d V \\
& -\int_{\partial \Omega_{0}} \overline{\boldsymbol{t}} \cdot \delta \boldsymbol{u} d \Gamma
\end{aligned}
$$

with $\boldsymbol{x}_{q}=\boldsymbol{x}_{0}+\boldsymbol{a}_{q}$. The load $\overline{\boldsymbol{n}}$ in this paper is the sum of the volume forces $\rho_{0} \overline{\boldsymbol{b}}$ and the surface loads $\overline{\boldsymbol{t}}$. The vector of stress resultants $\widehat{\boldsymbol{S}}$ can be expressed as following:

$$
\widehat{\boldsymbol{S}}=\left[\begin{array}{c}
\mathbf{F} \\
\mathbf{M}
\end{array}\right]=\left[\begin{array}{c}
F^{1} \\
F^{2} \\
F^{3} \\
M^{1} \\
M^{2} \\
\left.\begin{array}{c}
\text { normalforce } \\
\text { shearforce } \\
\text { shearforce } \\
M^{3}
\end{array}\right] \text { bendingmoment } \\
\text { bendingmoment }
\end{array}\right.
$$

By using the directional derivative we can easily linearize the weak form of Eq. (24). This yields

$$
\begin{aligned}
D \mathcal{G}(\boldsymbol{v}, \delta \boldsymbol{v}) \cdot \Delta \boldsymbol{v} & =\int_{s}\left(\delta \widehat{\boldsymbol{E}}^{T} \widehat{\boldsymbol{D}} \Delta \widehat{\boldsymbol{E}}+\Delta \delta \widehat{\boldsymbol{E}}^{T} \widehat{\boldsymbol{S}}\right) d S \\
& -\int_{s} \overline{\boldsymbol{q}} \cdot \Delta \delta \boldsymbol{x}_{q} d S
\end{aligned}
$$

where, $\quad \boldsymbol{v}=\left[\begin{array}{ll}\boldsymbol{u} & \boldsymbol{R}\end{array}\right]^{T}$ describes the independent kinematic quantities of the beam. In parallel with linear situation we can derive the discretization of the following kinematic variables by using the NURBS shape functions.

$$
\boldsymbol{X}_{0}=\sum_{I=1}^{n e n} N_{I}(\xi) \boldsymbol{X}_{I} \boldsymbol{x}_{0}=\sum_{I=1}^{n e n} N_{I}(\xi)\left(\boldsymbol{X}_{I}+\boldsymbol{u}_{I}\right)
$$

Thus, the virtual beam strains $\delta \hat{E}$ can be written as follows:

$$
\begin{aligned}
\delta \widehat{\boldsymbol{E}}=\sum_{I=1}^{n e n} \boldsymbol{B}_{I} \delta \boldsymbol{v}_{I} \boldsymbol{B}_{I} & \\
& =\left[\begin{array}{cc}
N_{I}^{\prime} \boldsymbol{R}_{I}^{T} & N_{I} \boldsymbol{B}_{\varepsilon I}^{T} \\
\mathbf{0} & N_{I}^{\prime} \boldsymbol{B}_{\kappa I}^{T}{ }^{\prime}+N_{I} \boldsymbol{B}_{\kappa I}^{T}
\end{array}\right]
\end{aligned}
$$

where, the matrix $\boldsymbol{B}_{\varepsilon I}, \boldsymbol{B}_{\kappa I}, \boldsymbol{B}_{\kappa I}^{\prime}$ are defined as follows:

$$
\begin{aligned}
\boldsymbol{B}_{\kappa I}:=\left[\begin{array}{lll}
\boldsymbol{O}_{2 I}\left(\boldsymbol{a}_{3}\right) & \boldsymbol{O}_{3 I}\left(a_{1}\right) & \boldsymbol{O}_{1 I}\left(\boldsymbol{a}_{2}\right)
\end{array}\right] \boldsymbol{B}_{\varepsilon I} \\
:=\left[\begin{array}{lll}
\boldsymbol{O}_{1 I}\left(\boldsymbol{x}_{0}^{\prime}\right) & \boldsymbol{O}_{2 I}\left(\boldsymbol{x}_{0}^{\prime}\right) & \boldsymbol{o}_{3 I}\left(\boldsymbol{x}_{0}^{\prime}\right)
\end{array}\right] \\
\boldsymbol{B}_{\kappa I}^{\prime}:=\left[\begin{array}{lll}
\boldsymbol{o}_{3 I}\left(\boldsymbol{a}_{2}^{\prime}\right) & \boldsymbol{o}_{1 I}\left(\boldsymbol{a}_{3}^{\prime}\right) & \boldsymbol{o}_{2 I}\left(\boldsymbol{a}_{1}^{\prime}\right)
\end{array}\right]
\end{aligned}
$$

with $\boldsymbol{O}_{m I}(\cdot)=\boldsymbol{a}_{m I} \times(\cdot)$.

Subsequently we insert the finite element interpolation into the linearized form (26) and we obtain

$$
\begin{gathered}
\mathcal{L}[\mathcal{G}(\boldsymbol{v}, \delta \boldsymbol{v})]=\bigcup_{e=1}^{\text {numel }} \sum_{I=1}^{\text {nen }} \sum_{K=1}^{\text {nen }} \delta \boldsymbol{v}_{I}^{T}\left(\boldsymbol{f}_{I}^{e}\right. \\
\left.+\boldsymbol{K}_{I K}^{e} \Delta \boldsymbol{v}_{K}\right)
\end{gathered}
$$

Hence the matrix $\boldsymbol{K}_{I K}^{e}$ describes the tangential stiffness matrix of element $e$ of the nodes $I$ and $\boldsymbol{K}$. The vector $\boldsymbol{f}_{I}^{e}$ is the sum of the internal and external nodal forces of the node I. Considering Eqs. (26)-(29) we obtain 


$$
\begin{array}{r}
\boldsymbol{K}_{I K}^{e}=\int_{S}\left(\boldsymbol{B}_{I}^{T} \widehat{\boldsymbol{D}} \boldsymbol{B}_{K}+\boldsymbol{G}_{I K}+\boldsymbol{P}_{I K}\right) d S \boldsymbol{f}_{I} \\
=\int_{S}\left(\boldsymbol{B}_{I}^{T} \widehat{\boldsymbol{s}}-N_{I} \overline{\boldsymbol{q}}\right) d S
\end{array}
$$

with $\overline{\boldsymbol{q}}=\left[\overline{\boldsymbol{n}}, \overline{\boldsymbol{m}}_{I}\right]^{T}$. In this paper the matrix $\widehat{\boldsymbol{D}}$ denotes the elasticity matrix, which can be described as follows:

$$
\widehat{\boldsymbol{D}}=\operatorname{diag}\left[E A, G A, G A, G I_{T}, E I_{33}, E I_{22}\right] .
$$

The matrix $\boldsymbol{G}_{I K}$ and $\boldsymbol{P}_{I K}$ come from the linearized virtual strains and external loading. The detail of those matrixes can be found in Ref. [9].

\section{Numerical Applications}

\subsection{Cantilever Timoshenko Beam}

In order to test the reliability of the Code we present two examples with finite deformations and elastic material behavior in this section. The first example is a cantilever Timoshenko-beam with a concentrated load acting downward at the free end, which is illustrated in Fig. 2.

The geometrical and material data of this Timoshenko-beam are given as follows:

$$
\ell=20 t=1 b=1 \mathrm{E}=1 \cdot 10^{7} v=0
$$

The maximal deflection of this beam can be expressed as follow:

$$
\omega_{\max }=\underbrace{\frac{F l^{3}}{3 E I}}_{\omega_{b}}+\underbrace{\frac{F l}{G A_{s}}}_{\omega_{s}}
$$

Now, we simulate the cantilever beam in linear and nonlinear situation with increasing load, respectively. The results are presented in Table 1.

The differences between the both beams are illustrated in Fig. 3. The deflections of nonlinear beam are always smaller than the deflections of linear beam. Those results match with our common sense.
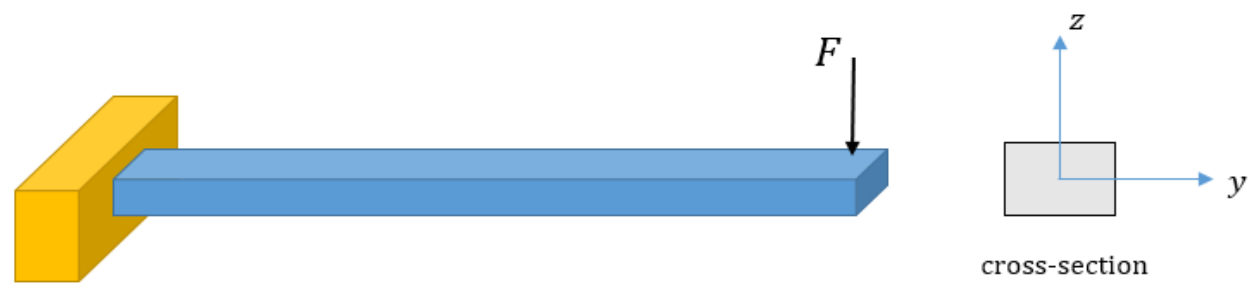

cross-section

Fig. 2 Cantilever Timoshenko-beam.

Table 1 Results: deflections of linear and nonlinear Timoshenko-beam.

\begin{tabular}{llll}
\hline Force & Linear beam & Nonlinear beam & Difference \\
\hline 100 & $3.21 \mathrm{E}-1$ & $3.20 \mathrm{E}-1$ & $1.00 \mathrm{E}-3$ \\
150 & $4.82 \mathrm{E}-1$ & $4.80 \mathrm{E}-1$ & $1.90 \mathrm{E}-3$ \\
200 & $6.42 \mathrm{E}-1$ & $6.39 \mathrm{E}-1$ & $3.40 \mathrm{E}-3$ \\
250 & $8.03 \mathrm{E}-1$ & $7.97 \mathrm{E}-1$ & $5.70 \mathrm{E}-3$ \\
300 & $9.64 \mathrm{E}-1$ & $9.55 \mathrm{E}-1$ & $8.90 \mathrm{E}-3$ \\
350 & $1.12 \mathrm{E}+0$ & $1.11 \mathrm{E}+0$ & $1.32 \mathrm{E}-2$ \\
400 & $1.28 \mathrm{E}+0$ & $1.27 \mathrm{E}+0$ & $1.87 \mathrm{E}-2$ \\
450 & $1.45 \mathrm{E}+0$ & $1.42 \mathrm{E}+0$ & $2.56 \mathrm{E}-2$ \\
500 & $1.61 \mathrm{E}+0$ & $1.57 \mathrm{E}+0$ & $3.40 \mathrm{E}-2$ \\
550 & $1.77 \mathrm{E}+0$ & $1.72 \mathrm{E}+0$ & $4.41 \mathrm{E}-2$ \\
600 & $1.93 \mathrm{E}+0$ & $1.87 \mathrm{E}+0$ & $5.60 \mathrm{E}-2$ \\
650 & $2.09 \mathrm{E}+0$ & $2.02 \mathrm{E}+0$ & $6.99 \mathrm{E}-2$ \\
700 & $2.25 \mathrm{E}+0$ & $2.16 \mathrm{E}+0$ & $8.57 \mathrm{E}-2$ \\
\hline
\end{tabular}




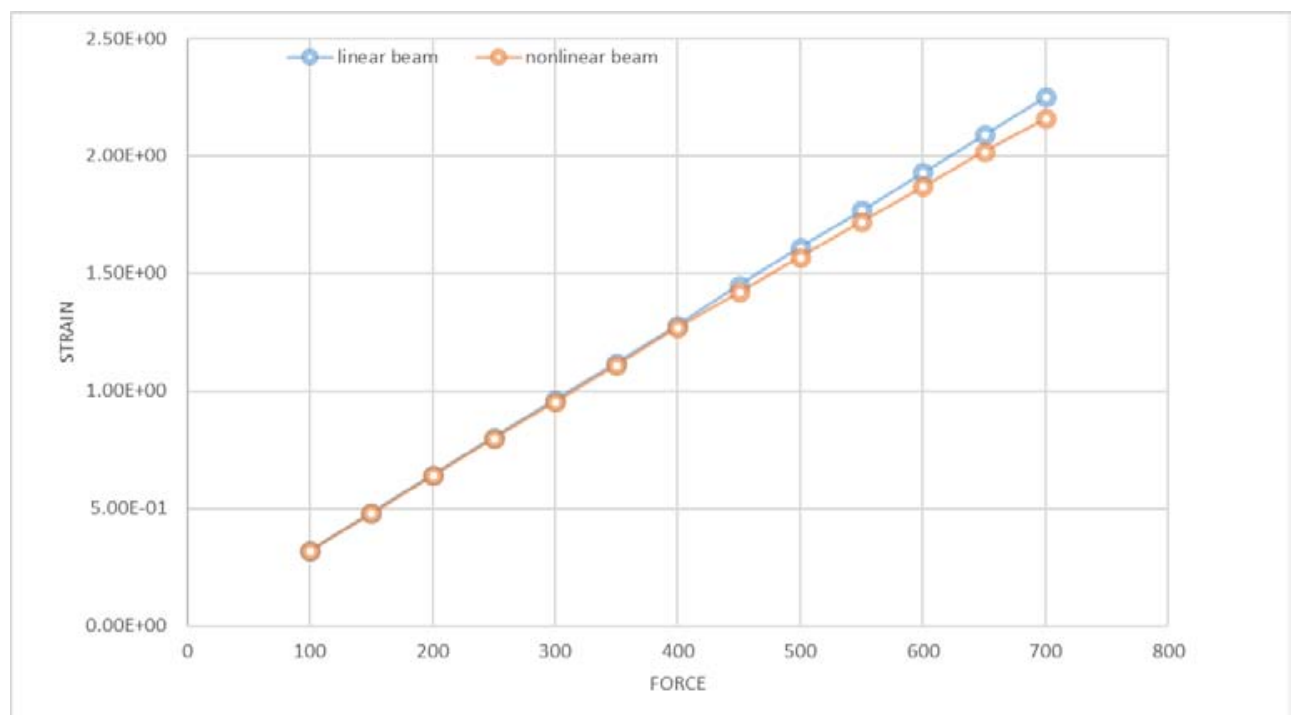

Fig. 3 Deflection between linear and nonlinear Timoshenko-beam.

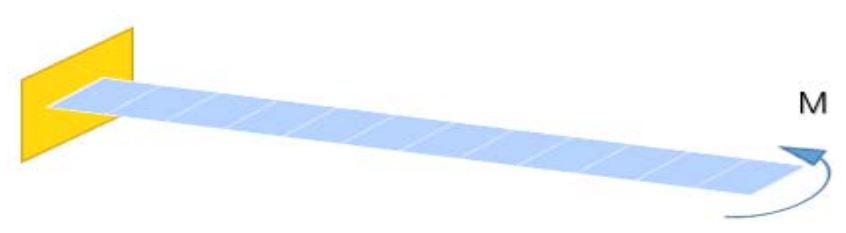

Fig. 4 Cantilever subjected to end moment.

Table 2 Result: deflection of cantilever.

\begin{tabular}{|l|l|l|l|}
\hline Grad & M & Analytical & Numerical \\
\hline 6.88 & 1 & 11.94 & 11.94 \\
\hline 10.32 & 1.5 & 11.87 & 11.87 \\
\hline 13.76 & 2 & 11.78 & 11.78 \\
\hline 17.2 & 2.5 & 11.66 & 11.66 \\
\hline 20.64 & 3 & 11.52 & 11.52 \\
\hline 27.5 & 4 & 11.19 & 11.19 \\
\hline 34.4 & 5 & 10.81 & 10.81 \\
\hline 41.3 & 6 & 10.41 & 10.41 \\
\hline 48.2 & 7 & 9.989 & 9.989 \\
\hline 55 & 8 & 9.572 & 9.572 \\
\hline
\end{tabular}

\subsection{Cantilever Subjected to End Moment}

With the second example we investigate the roll-up of a cantilevered flat plate strip, which is illustrated in Fig. 4. The plate strip has a wall thickness $t=$ 0.1 with the length $\ell=12$ and width $b=1$. The Young's modulus $E$ is $1.2 \cdot 10^{6}$. Hence, we set the Poisson ratio to zero in order to make a beam-like behavior.

The deflection of the plate strip can be expressed as

$$
\begin{gathered}
u(M)=\left[\sin \left(M / M_{0}\right) \cdot M_{0} / M-1\right] \cdot \text { LwithM }_{0} \\
=E I / L
\end{gathered}
$$

Eq. (35) can be found in Ref. [10]. The numerical solutions are reported in Table 2, which have no difference from the analytical solutions.

\section{Conclusions}

In this paper an efficient and robust isogeometric linear and nonlinear cantilever formulation has been presented. The main contribution of this work is programing an efficient code in order to analyze the deflection of the cantilever. Two numerical examples by using the isogeometric analysis have been discussed. The results have been calculated by computer and presented in the end. Because of the distinguished characters of the NURBS functions the accurate results can be obtained. In comparison with the standard lagrange shape function the NURBS functions have distinct advantages. The computational cost and the time of work can be spared by using isogeometric analysis. This shows high significance for complex finite element analysis in CAD.

\section{References}

[1] Timoshenko, S. P. 1921. "On the Correction Factor for Shear of the Differential Equation for Transverse Vibrations of Bars of Uniform Cross-section.” Philosophical Magazine, 744.

[2] Bathe, K. J., and Bolourchi, S. 1979. "Large Displacement Analysis of Three-Dimensional Beam 
Structures.” Int. J. Num. Meth. Engng. 14: 961-86.

[3] Benson, D., Gambarin, F. I., Hartmann, S., Hughes, T. J. R., Reali, A., Morganti, S., and Auricchio, F. 2014. Patient-Specific Isogeometric Structural Analysis of Aortic Valve Closure. Technical report, The Institute for Computational Engineering and Sciences.

[4] Wayne Tiller Les Piegl. 1997. The NURBS Book (2nd ed.). New York: Springer.

[5] Bazilevs, Y., Austin Cottrell, J., and Hughes, T. J. R. 2009. Isogeometric Analysis: Toward integration of $C A D$ and FEA. New Jersey: Wiley.

[6] Schroeder, J., Wall, W. A., Gross, D., and Hauger, H. 2017. Technische Mechanik 2: Elastostatik. New York: Springer.

[7] Reissner, E. 1981. “On Finite Deformations of
Space-Curved Beams.” J. Appl. Math. Phys. (ZAMP) 32: 734-44.

[8] Gruttmann, F., Sauer, R., and Wagner, W. 1998. “A Geometrical Nonlinear Eccentric 3D-Beam Element with Arbitrary Cross-Sections.” Comp. Meth. Appl. Mech. Engrg. 160: 383-400.

[9] Gruttman, F., Sauer, R., and Wagner, W. 2000. "Theory and Numerics of Three-Dimensional Beams with Elastoplastic Material Behavior.” Int. J. Numer. Meth. Engnr. 48: 1675-702.

[10] Dornisch. W., Müller, R., and Klinkel, S. 2016. “An Efficient and Robust Rotational Formulation for Isogeometric Reissner-Mindlin Shell Elements.” Comput. Methods Appl. Mech. Engrg. 303: 1-34. 